\title{
APPROXIMATE DUAL AND APPROXIMATE VECTOR VARIATIONAL INEQUALITY FOR MULTIOBJECTIVE OPTIMIZATION
}

\author{
G.-Y. CHEN and B. D. CRAVEN
}

(Received 12 April 1988; revised 4 July 1988)

\begin{abstract}
An approximate dual is proposed for a multiobjective optimization problem. The approximate dual has a finite feasible set, and is constructed without using a perturbation. An approximate weak duality theorem and an approximate strong duality theorem are obtained, and also an approximate variational inequality condition for efficient multiobjective solutions.
\end{abstract}

1980 Mathematics subject classification (Amer. Math. Soc.) (1985 Revision): 90 C 31.

\section{Introduction}

Consider the finite dimensional linear space $\mathbf{R}^{p}$, equipped with a partial ordering $\geq_{s}$, defined by a closed pointed convex cone $S$, with interior int $S \neq \varnothing$.

Definition 1 (see [3]). For a given $e \in S$, a point $c \in A \subset \mathbf{R}^{p}$ is said to be an $e$-[weak] minimum of $A$ if there exists no $x \in A$ satisfying $0 \neq c-x-e \in S$ [ $\in$ int $S$ ]. A point $c \in A$ is said to be an $e$-[weak] maximum if there exists no $x \in A$ such that $0 \neq x-c-d \in S[\in$ int $S$ ]. When $e=0$, an $e$-[weak] minimum (e-[weak] maximum) is said to be a [weak] minimum ([weak] maximum). A set $W$ is said to $e$-upper dominate a set $V$ if

$$
(\forall v \in V)(\exists w \in W) w+e-v \in S .
$$

A set $W$ is said to $e$-strongly upper dominate a set $V$ if

$$
(\forall v \in V)(\exists w \in W) w+e-v \in \operatorname{int} S .
$$

(C) 1989 Australian Mathematical Society $0263-6115 / 89 \$ A 2.00+0.00$ 
The order-interval $[-e, e]:=\left\{x \in \mathbf{R}^{p}: e \geq_{s} \quad x \geq_{s}-e\right\}$. Denote its interior by $(-e, e)$. Denote by $\mathbf{R}^{p \times m}$ the space of all $p \times m$ real matrices $\Lambda$, and by $\|\Lambda\|$ the norm of $\Lambda$ in this space. Let $D \subset \mathbf{R}^{m}$ be a closed convex cone. Let $M:=\left\{\Lambda \in \mathbf{R}^{p \times m}:\|\Lambda\|=1, \Lambda(D) \subset S\right\}$. Then $M$ is compact.

A function $h: C \rightarrow \mathbf{R}^{p}$ is $S$-convex if $C \subset \mathbf{R}^{n}$ is convex, and

$$
(\forall x, y \in C, \forall \alpha \in(0,1)) \quad h(\alpha x+(1-\alpha) y) \leq s \alpha h(x)+(1-\alpha) h(y) .
$$

Consider a nonlinear multiobjective optimization problem:

WMin $f(x)$ subject to $x \in X:=\{x \in E,-g(x) \in D\}$,

where $f: \mathbf{R}^{n} \rightarrow \mathbf{R}^{p}, g: \mathbf{R}^{n} \rightarrow \mathbf{R}^{m}$ are vector functions, $E \subset \mathbf{R}^{n}$, and weak minimum (WMin; and WMax later) are as in Definition 1.

Definition 2. A vector valued Lagrangian for $(\mathrm{P})$ is $L(x, \Lambda):=f(x)+$ $\Lambda g(x)$.

Following Sawaragi, Nakayama and Tanino [1], it will be assumed that

(i) $E$ is nonempty compact,

(ii) $f$ is continuous, and

(iii) $g$ is continuous.

Under these assumptions, it is readily shown that $X$ and $f(X)$ are compact.

Definition 3. The dual map $\Phi: M \rightarrow \mathbf{R}^{p}$ is defined by

$$
\Phi(\Lambda):=W M i n ~ L(E, \Lambda),
$$

for each $\Lambda \in M$. A dual problem [1] to $(\mathrm{P})$ is

$\operatorname{WMax} \bigcup_{\Lambda \in M} \Phi(\Lambda)$.

LEMmA 1. Under the above assumptions, for each $\Lambda \in M$, the $\operatorname{sets} L(E, \Lambda)$ and $\Phi(\Lambda)$ are compact.

Proof. Since $E$ is compact, and $f$ and $g$ are continuous, $L(E, \Lambda)$ is compact, for each $\Lambda \in M$. Hence $\Phi(\Lambda)$ is nonempty and bounded. Consider a sequence $\left\{y_{k}\right\} \subset \Phi(\Lambda),\left\{y_{k}\right\} \rightarrow y_{0}$. If $y_{0} \notin \Phi(\Lambda)$ then there exists $v \in L(E, \Lambda)$ such that $y_{0}-v \in$ int $S$. Since $\left\{y_{k}\right\} \rightarrow y_{0}$, there exists $k_{0} \in N$ such that $y_{k}-v \in \operatorname{int} S$ when $k \geq k_{0}$, contradicting the weak minimum. So $\Phi(\Lambda)$ is closed, and therefore compact.

LeMma 2. The map $\Phi: M \rightarrow \mathbf{R}^{p}$ is upper semicontinuous, and $\Phi(M)$ is compact.

Proof. Let $\left\{\Lambda_{k}\right\} \rightarrow \Lambda_{0}$ in $M,\left\{v_{k}\right\} \rightarrow v_{0},(\forall k) v_{k} \in L\left(E, \Lambda_{k}\right)$. For each $k$, there exists $x_{k} \in E$ such that $v_{k}=L\left(x_{k}, \Lambda_{k}\right)$. Since $E$ is compact, there 
is a subsequence $\left\{x_{k_{j}}\right\} \subset\left\{x_{k}\right\}$ such that $\left\{x_{k_{j}}\right\} \rightarrow x_{0} \in E$. Since $f$ and $g$ are continuous, $\left\{v_{k_{j}}\right\} \rightarrow L\left(x_{0}, \Lambda_{0}\right)=v_{0} \in L\left(E, \Lambda_{0}\right)$. Thus the mapping $L(E, \cdot)$ is upper semicontinuous on $M$. To show also lower semicontinuity, let $\left\{\Lambda_{k}\right\} \rightarrow \Lambda_{0}$ in $M$, and let $v_{0} \in L\left(E, \Lambda_{0}\right)$; thus $v_{0}=L\left(x_{0}, \Lambda_{0}\right)$ for some $x_{0} \in E$; then $\left\{v_{k}\right\}:=\left\{L\left(x_{0}, \Lambda_{k}\right)\right\} \rightarrow v_{0}$, and $(\forall k) v_{k} \in L\left(E, \Lambda_{k}\right)$.

Let $\left\{\Lambda_{k}\right\} \rightarrow \Lambda_{0}, \varphi_{k} \in \Phi\left(\Lambda_{k}\right)$ for each $k,\left\{\varphi_{k}\right\} \rightarrow \varphi_{0}$. If $\varphi_{0} \notin \Phi\left(\Lambda_{0}\right)$ then $\varphi_{0}-v_{0} \in$ int $S$ for some $v_{0} \in L\left(E, \Lambda_{0}\right)$. Since $\left\{\varphi_{k}\right\} \rightarrow \varphi_{0}$, there exists a $k_{0}>0$ such that for each $k \geq k_{0} \varphi_{k}-v_{0} \in$ int $S$. Since $L(E, \cdot)$ is continuous at $\Lambda_{0}$, for each $k$ there exists $v_{k} \in L\left(E_{k}\right)$ such that $\left\{v_{k}\right\} \rightarrow v_{0}$, thus $\varphi_{k}-v_{k} \in \operatorname{int} S$, and thus $\varphi_{k} \notin$ WMin $L\left(E, \Lambda_{k}\right)$. The contradiction shows that $\Phi$ is upper semicontinuous. Since $M$ is compact, $\Phi(M)$ is compact [6].

REMARK. The conclusions of Lemma 1 and Lemma 2 still hold if $\Phi(\Lambda)$ is changed to $e-$ WMin $L(E, \Lambda)$.

\section{Approximate dual}

We now introduce an approximate dual for the multiobjective problem (P). No perturbation map is required.

Lemma 3. Let $A \subset \mathbf{R}^{p}$ be compact, and $e \in \operatorname{int} S$. Then there exists a finite subset $K \subset A$ such that $K$ e-strongly upper dominates $A$.

Proof. The family of open order intervals $\{(a-e, a+e): a \in A\}$ covers $A$, so there exists a finite subfamily $\left\{\left(a_{i}-e, a_{i}+e\right): i=1,2, \ldots, k\right\}$ which covers $A$. Let $K=\left\{a_{1}, a_{2}, \ldots, a_{k}\right\}$. If $a \in A$, then $a \in\left(a_{i}-e, a_{i}+e\right)$ for some $i$, and $a_{i}+e-a \in \operatorname{int} S$; thus $K \varepsilon$-strongly upper dominates $A$.

REMARK. Similar results hold with upper replaced by lower.

Since $f$ is continuous and $X$ is compact, $f(X)$ is compact. By Lemma 3, there exists a finite subset $U \subset f(X)$, such that $U e$-strongly lower dominates $f(X)$, and thus $(\forall v \in f(X))(\exists u \in U) u-e-v \in-\operatorname{int} S$. So a primal approximate problem may be defined as

WMin $U$.

For each $\Lambda \in M$, define $\Phi^{*}(\Lambda)$ to be the set of $e$-weak minima of $L(E, \Lambda)$. Then $\Phi^{*}(\Lambda) \neq \varnothing$. Set $Q:=\bigcup_{\Lambda \in M} \Phi^{*}(\Lambda)$. By Lemma 2 , the set $Q$ is compact. By Lemma 3, there exists a finite subset $W \subset Q$ such that $(\forall q \in Q)(\exists w \in W)$ $w-e-q \in-S$. So an approximate dual problem may be defined as

WMax $W$.

Two obvious corollaries follow. 
COROllary 1. If $u_{0}$ is a weak minimum of $\left(\mathrm{P}^{*}\right)$, then $u_{0}$ is an e-weak minimum of $(\mathrm{P})$.

Proof. If not, then there exists $v \in f(X)$ such that $u_{0}-v-e \in \operatorname{int} S$. But, given $v \in f(X)$, there exists $u^{\prime} \in U$ with $u^{\prime}-v-e \in-$ int $S$. Combining these, $u_{0}-u^{\prime} \in$ int $S$, contradicting the weak minimum.

COROLlary 2. If $w_{0}$ is a weak maximum of $\left(\mathrm{D}^{*}\right)$ then $w_{0}$ is an e-weak maximum of $\left(\mathrm{D}_{\mathrm{TS}}\right)$.

Proof. If not, then there exists $v \in \Phi^{*}(M)$ such that $v-\left(w_{0}+e\right)=\operatorname{int} S$. Given this $v$, there exists $w^{\prime} \in W$ such that $w^{\prime}+e-v \in \operatorname{int} S$. Combining these, we obtain $w^{\prime}-w_{0} \in$ int $S$, contradicting the weak maximum.

THEOREM 1 (APPROXIMATE WEAK DUALITY). For each $u \in U$ and each $w \in W, w-u-e \notin S \backslash\{0\}$.

Proof. If $w \in W$, then $w \in \Phi^{*}(\Lambda)$ for some $\Lambda \in M$. Then $(\forall x \in X) w-e-[f(x)+\Lambda g(x)] \in H:=\mathbf{R}^{p} \backslash \operatorname{int} S$.

TheOREM 2 (APPROXIMATE STRONG DUALITY). Let $x^{*} \in X$ and $\Lambda^{*} \in M$ satisfying $w^{*}:=f\left(x^{*}\right) \in \Phi^{*}\left(\Lambda^{*}\right) \cap U \cap W$. Then $x^{*}$ is an e-weak minimum of $f(X)$, and $w^{*}$ is an e-weak maximum of $\Phi(M)$.

Proof. If $x^{*}$ is not an $e$-weak minimum of $f(X)$, then there exists $x \in X$ such that $f\left(x^{*}\right)-e-f(x) \in$ int $S$. Since $f\left(x^{*}\right) \in W$ and $\Lambda^{*} g\left(x^{*}\right) \in-S$, it follows that $f\left(x^{*}\right)-e-\left[f(x)+\Lambda^{*} g(x)\right] \in$ int $S$, contradicting $f\left(x^{*}\right) \in$ $\Phi^{*}\left(\Lambda^{*}\right)$.

If $w^{*}=f\left(x^{*}\right)$ is not an $e$-weak maximum of $\Phi(M)$, there is $w \in \bigcup_{\Lambda \in M} \Phi(\Lambda)$ such that $w-f\left(x^{*}\right)-e \in \operatorname{int} S$. Now $w=\Phi(\Lambda)$ for some $\Lambda \in M$. Since $\Lambda g\left(x^{*}\right) \in-S, w-\left[f\left(x^{*}\right)+\Lambda g\left(x^{*}\right)+e\right] \in$ int $S$, and hence $w$ is not an $e$-weak maximum of $L(E, \Lambda)$.

\section{Approximate vector variational inequality}

Let $C \in \mathbf{R}^{n}$ be a nonempty convex set, $S \subset \mathbf{R}^{p}$ a closed convex cone with int $S \neq \varnothing$, and $f: C \rightarrow \mathbf{R}^{p}$ a vector valued function. Denote by $\mathbf{L}\left(\mathbf{R}^{n}, \mathbf{R}^{p}\right)$ the space of all linear mappings from $\mathbf{R}^{n}$ into $\mathbf{R}^{n}$. Let $G: C \rightarrow \mathbf{L}\left(\mathbf{R}^{n}, \mathbf{R}^{p}\right)$ be a mapping. A generalized vector variational inequality is the problem of finding $x_{0} \in C$ and $A \in G\left(x_{0}\right)$ such that

$$
(\forall x \in C) A\left(x-x_{0}\right) \notin-\operatorname{int} S .
$$


TheOREM 3. Let $C \subset \mathbf{R}^{n}$ be compact convex; let $S \subset \mathbf{R}^{p}$ be a closed convex pointed cone with int $S \neq \varnothing$; let $f: C \rightarrow \mathbf{R}^{p}$ be $S$-convex, (Fréchet) continuously differentiable at $x_{0} \in C$, and differentiable on $C$; let $x_{0}$ be a weak minimum of $f(x)$ subject to $x \in C$. Then, for each $e \in$ int $S$, there exists a finite subset $W \subset C$ such that $(\forall w \in W) f^{\prime}\left(x_{0}\right)\left(w-x_{0}\right)+e \notin-\operatorname{int} S$.

Proof. Since $C$ is a compact convex, and $f^{\prime}\left(x_{0}\right)$ is a continuous linear mapping, $K:=f^{\prime}\left(x_{0}\right)\left(C-x_{0}\right)$ is compact convex. By Lemma 3 , for each $e \in$ int $S$, there exists a finite subset $W^{\prime} \subset C$ such that, for each $x \in C$, there is $w_{x} \in W^{\prime}$ such that $\left[f^{\prime}\left(x_{0}\right)\left(w_{x}-x_{0}\right)+e\right]-f^{\prime}\left(x_{0}\right)\left(x-x_{0}\right) \in S$. Denote by $W$ the finite subset $\left\{w_{x} \in W^{\prime}: x \in C\right\}$. From [2, Theorem 4], since $f$ is $S$-convex, $(\forall x \in C) f^{\prime}\left(x_{0}\right)\left(x-x_{0}\right) \notin-$ int $S$. Let $Q:=\mathbf{R}^{p} \backslash(-$ int $S)$. Then $f^{\prime}\left(x_{0}\right)\left(w_{x}-x\right)+e \in S+Q \subset Q$. Hence $(\forall w \in W) f^{\prime}\left(x_{0}\right)\left(w-x_{0}\right)+e \notin-i n t S$.

Since $f^{\prime}(x)(C-x)$ is compact convex, for each $x \in C$, Lemma 3 shows that, for each $x \in C$ and each $e \in$ int $S$, there exists a finite subset $M(x) \subset$ $f^{\prime}(x)(C-x)$ such that $\left(\forall m \in f^{\prime}(x)(C-x)\right)\left(\exists m^{\prime} \in M(x)\right) m-\left[m^{\prime}-e\right] \in S$. For each $x \in C$ and each $m^{\prime} \in M(x)$, let $u \equiv u\left(x, m^{\prime}\right)$ be an element of $C$ such that $f^{\prime}(x)(u-x) \in M(x)$; denote by $U(x)$ the finite set $\left\{u\left(x, m^{\prime}\right): m^{\prime} \in\right.$ $M(x)\}$.

Consider now the generalized inequality system, for $x \in C$ :

$$
(\forall u \in U(x)) f^{\prime}(x)(u-x)-e \notin-\operatorname{int} S .
$$

THEOREM 4. Let $f: C \rightarrow \mathbf{R}^{p}$ be $S$-convex and continuously differentiable on $C$, where $C \subset \mathbf{R}^{n}$ is a compact convex set; let $e \in$ int $S$. If $x_{0}$ is a solution (GI), then $x_{0}$ is a weak minimum of $f(x)$ subject to $x \in C$.

Proof. Since $f$ is $S$-convex and differentiable, $(\forall x \in C) f(x)-f\left(x_{0}\right)-$ $f^{\prime}\left(x_{0}\right)\left(x-x_{0}\right) \in S$. Since $f^{\prime}(x)(C-x)$ is compact, Lemma 3 shows that, for each $x \in C$, there exists $u \in U\left(x_{0}\right)$ such that

$$
f^{\prime}\left(x_{0}\right)\left(x-x_{0}\right)-\left[f^{\prime}\left(x_{0}\right)\left(u-x_{0}\right)-e\right] \in S .
$$

Since $x_{0}$ is a solution of $(\mathrm{GI}), f^{\prime}\left(x_{0}\right)\left(u-x_{0}\right) \in Q$, where $Q:=\mathbf{R}^{p} \backslash(-\operatorname{int} S)$. Adding these inclusions, $f(x)-f\left(x_{0}\right) \in S+S+Q \subset Q$. Thus $x_{0}$ is a weak minimum of $f(x)$ subject to $x \in C$.

\section{Acknowledgement}

The authors thank a referee for careful checking and useful comments. 


\section{References}

[1] Y. Sawaragi, H. Nakayama and T. Tanino, Theory of Multiobjective Optimization, (Math. Sci. Engrg., vol. 176, Academic Press, New York, 1985, 137-138).

[2] G.-Y. Chen and B. D. Craven, 'A vector variational inequality and optimization over an efficient set' $Z$. Oper. Res. (to appear).

[3] P. Loridan, ' $\varepsilon$-solutions in vector minimization problems', J. Optim. Theory Appl. 43 (1984), 265-276.

[4] J. Vályi, 'Approximate saddle-point theorems in vector optimization', J. Optim. Theory Appl. 55 (1987), 436-448.

[5] P. L. Yu, 'Cone convexity, cone extreme points, and nondominated solutions in decision problems with multiobjectives', J. Optim. Theory Appl. 14 (1974), 318-323.

[6] C. Berge, Topological Spaces, (Macmillan, New York, 1963).

Academia Sinica

Institute of Systems Science

Beijing 100080

China
Mathematics Department University of Melbourne Parkville, Victoria 3052

Australia 